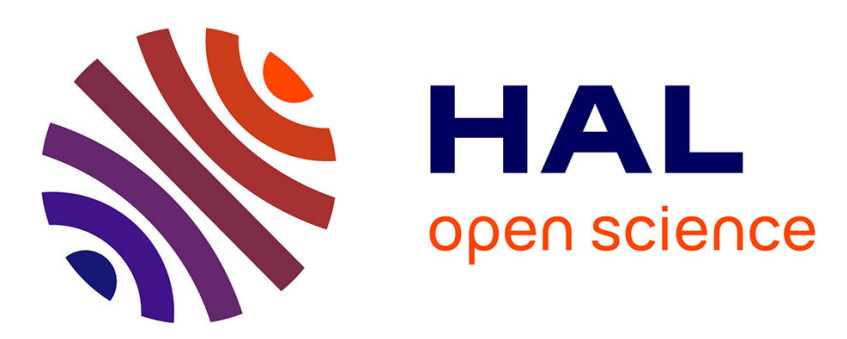

\title{
An acoustic model for automatic control of a slide flute
} Brigitte d'Andréa-Novel, Benoît Fabre, Jean-Michel Coron

\section{To cite this version:}

Brigitte d'Andréa-Novel, Benoît Fabre, Jean-Michel Coron. An acoustic model for automatic control of a slide flute. Acta Acustica united with Acustica, 2010, 96, pp.713-721. hal-00923617

\section{HAL Id: hal-00923617 \\ https://hal-mines-paristech.archives-ouvertes.fr/hal-00923617}

Submitted on 3 Jan 2014

HAL is a multi-disciplinary open access archive for the deposit and dissemination of scientific research documents, whether they are published or not. The documents may come from teaching and research institutions in France or abroad, or from public or private research centers.
L'archive ouverte pluridisciplinaire HAL, est destinée au dépôt et à la diffusion de documents scientifiques de niveau recherche, publiés ou non, émanant des établissements d'enseignement et de recherche français ou étrangers, des laboratoires publics ou privés. 


\title{
An acoustic model for automatic control of a slide flute
}

\author{
Brigitte d'Andréa-Novel, Benoît Fabre, Jean-Michel Coron
}

\begin{abstract}
In this paper, we consider the problem of modeling and control of a slide flute : a kind of recorder without finger holes but which is ended by a piston mechanism to modify the length of the resonator. A previous study has been done (see [3]), but with a very simple boundary condition for the mouth, corresponding to an ideal situation assuming that the acoustic pressure is zero at the entrance of the resonator.

In this work, we have taken into account a more realistic model, describing the coupling effects between the jet and the pipe. The jet is obtained by blowing through a flue channel and formed by flow separation at the flue exit, and finally directed towards a sharp edge, called the labium. The resulting structure can then be seen as a nonlinear oscillator coupled with the pipe which is a linear acoustic resonator. The pressure obtained through this model has been compared to the pressure measured on an actual instrument, a recorder closed at its end.

A modal analysis is then performed using the linearized boundary conditions which can also be used to compute the suitable blowing pressure and the suitable pipe length to obtain a desired fundamental frequency or equivalently a desired pitch. This will constitute the basis of our control algorithm.

A possible musical application of such a device is to build a flue instrument with a pitch independent of the dynamical level.

PACS number : 43.75
\end{abstract}

\section{INTRODUCTION}

Slide flutes are mostly used for jazz and popular music, even if they sometimes appear in the classical orchestra in works like the opera by Maurice Ravel, L'Enfant et les Sortilèges. We are interested in this paper to model and control this kind of instrument made of a cylindrical stopped resonator similar to a stopped organ pipe and of a blowing mouthpiece analogous to that of a recorder. Contrary to flutes, organ pipes and recorders, the variation of the pitch is obtained through a piston mechanism. From a physical point of view, stopped pipes have not been studied so widely as open pipes. Moreover, moving boundary conditions introduce interesting problems also from a mathematical point of view.

In the case of open pipes, physical models for the excitation mechanism have been developed to produce quite realistic sound synthesis (see e.g. [8], [19]). In the present work, we use the same model of the excitator and couple it to a pipe model with moving boundary conditions. Even if the

This work was partly supported by the french ANR project "CONSONNES"

Brigitte d'Andréa-Novel is with Mines ParisTech, Centre de Robotique, 60 bvd Saint-Michel, 75272 Paris Cedex 06, FRANCE brigitte.dandrea-novel@mines-paristech. fr

Benoît Fabre is with Université Pierre et Marie Curie, Lutherie, Acoustique, Musique, IJLRA, 11 rue de Lourmel, 75015 Paris, FRANCE benoit.fabre@upmc.fr

Jean-Michel Coron is with Université Pierre et Marie Curie and Institut Universitaire de France, B.C. 187, 4 place Jussieu, 75252 Paris Cedex 05, FRANCE coron@ann.jussieu.fr resulting dynamical model can be used for sound synthesis purposes (as illustrated in section VII), our main goal is to develop control algorithms to automatically control such physical instruments. An important musical application for such device is to produce musical dynamics (piano e forte) at constant pitch by adjusting both the blowing pressure and the slide position.

The obtention of physical models for analysis and synthesis for flue musical instruments such as organs or recorders has been an important research subject for a few decades. We will not be exhaustive, but we can mention the paper by Cremer and Ising [7] giving a first quasi-stationary model of the jet drive, which has been later improved by many authors (see e.g. [6], [11]). The works of Howe [12] pointed out the importance of vortex shedding at the labium. In fact, in steady blowing conditions, models not taking into account this effect (e.g. in [11]) led to an overestimation of the amplitude of the pressure oscillation in the pipe (see for example [17]). Therefore, as in [20], we have taken into account these interactions jet/labium, but as already mentioned, the system we are studying is different : the resonator's length is time-varying, controlled through the piston mechanism and there is no finger hole (see Fig. 1). The whole structure can then be described by two linear Partial Differential Equations coupled with nonlinear Ordinary Differential Equations describing the boundary conditions :

- for the mouth, taking into account the jet dynamics,

- and for the piston.

In section II we recall our pipe model. In section III, we give the physical models of the jet channel and the mouth. In section IV we compute the boundary condition at the end of the resonator and at its entrance, taking into account the jet dynamics. In section $\mathrm{V}$ we present the modal analysis we have developed from the linearized boundary conditions, and we compute the different frequencies which are functions of the resonator's length and of the steady blowing pressure. It can be noticed that the frequencies are odd multiples of the fundamental one, as expected for open-closed pipes instruments like the pan flute or the clarinet. Conversely, if we want to obtain a reference fundamental frequency, we can use the previous linearized boundary conditions, where we fix the desired frequency value, to compute the suitable blowing pressure and the suitable length. This will constitute our control algorithm described in section VI. Finally in section VII we present our numerical scheme together with some simulation results. Let us point out that in our previous paper [4], simulation results showed that the resulting blowing pressure range was lower than in actual pipes. This was mainly due to the simplifications 
of the jet and source models (see also [5, Sec. 3]) which overestimated the jet amplification and sound production at low jet velocities. To solve this problem, we propose to implement a numerical filter to obtain a more realistic dynamics of the jet position in the mouth. The resulting pressure is compared with experimental data obtained from an actual instrument and we conclude.
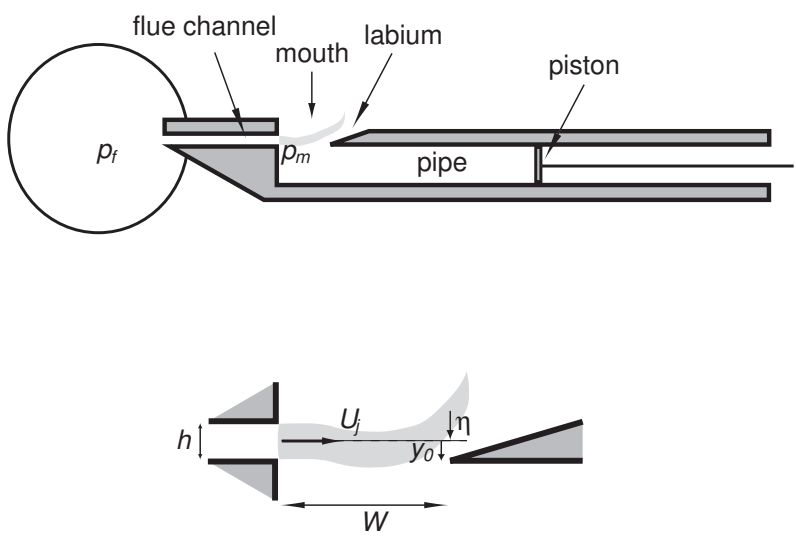

Fig. 1. The slide flute

\section{PHySICAL MODEL OF THE PIPE}

If $\rho_{0}$ denotes the air density at rest, $S_{p}$ the constant section of the pipe which is supposed to be cylindrical, and assuming the flow rate $u(x, t)$ at time $t$ and point $x$ in the pipe and the relative pressure $p(x, t)=P-P_{a t m}\left(P_{a t m}\right.$ denoting the atmospheric pressure) are uniform on a section, the Euler equation, giving the fluid dynamical properties can be written :

$$
\frac{\partial u}{\partial t}=-\frac{S_{p}}{\rho_{0}} \frac{\partial p}{\partial x}
$$

neglecting the viscous and thermal effects near the walls.

The mass conservation law has the following form :

$$
\frac{\partial \rho}{\partial t}=-\frac{\rho_{0}}{S_{p}} \frac{\partial u}{\partial x}
$$

Finally, assuming that the transformation is adiabatic, we have the following equation, $c$ being the sound velocity in the fluid :

$$
p=c^{2} \rho
$$

which allows to link the pressure fluctuations $p$ and the density fluctuations $\rho$.

Then, replacing $\rho$ from $p$ in (2), we obtain the second state equation which completes (1), i.e. :

$$
\frac{\partial p}{\partial t}=-\frac{\rho_{0} c^{2}}{S_{p}} \frac{\partial u}{\partial x}
$$

Differentiating (4) with respect to $t$, (1) with respect to $x$ and collecting the resulting equations lead to the d'Alembert equation :

$$
\frac{\partial^{2} p}{\partial t^{2}}-c^{2} \frac{\partial^{2} p}{\partial x^{2}}=0
$$

Equations (1) and (4) allow one to write the system dynamics in the following state-space form with $Y=\left(\begin{array}{l}u \\ p\end{array}\right)$ :

$$
\frac{\partial Y}{\partial t}+A \frac{\partial Y}{\partial x}=0, \text { with } A=\left(\begin{array}{cc}
0 & S_{p} / \rho_{0} \\
\rho_{0} c^{2} / S_{p} & 0
\end{array}\right) .
$$

This representation can be diagonalized :

$$
\partial_{t} \xi+\Lambda \partial_{x} \xi=0, \text { with } \Lambda=\left(\begin{array}{cc}
c & 0 \\
0 & -c
\end{array}\right)
$$

where the change of coordinates is given by :

$$
\xi=\left(\begin{array}{c}
\alpha \\
\beta
\end{array}\right)=\left(\begin{array}{c}
u+\frac{S_{p}}{\rho_{0} c} p \\
u-\frac{S_{p}}{\rho_{0} c} p
\end{array}\right)
$$

and

$$
Y=\left(\begin{array}{c}
u \\
p
\end{array}\right)=\left(\begin{array}{c}
\frac{\alpha+\beta}{2} \\
\frac{\rho_{0} c(\alpha-\beta)}{2 S_{p}}
\end{array}\right) .
$$

The eigenvalues $c>0$ and $-c<0$ being respectively the velocity of the ingoing wave $\alpha(x, t)$ and of the outgoing wave $\beta(x, t) . \alpha(x, t)$ and $\beta(x, t)$ satisfy two classical wave equations :

$$
\begin{gathered}
\frac{\partial \alpha}{\partial t}+c \frac{\partial \alpha}{\partial x}=0 \text { and } \\
\frac{\partial \beta}{\partial t}-c \frac{\partial \beta}{\partial x}=0 .
\end{gathered}
$$

The quantities $\frac{\partial \alpha}{\partial t}+c \frac{\partial \alpha}{\partial x}$ and $\frac{\partial \beta}{\partial t}-c \frac{\partial \beta}{\partial x}$ can be seen as the time derivatives $\frac{d \alpha}{d t}$ and $\frac{d \beta}{d t}$ of $\alpha$ and $\beta$ in $(x, t)$ along the solutions of :

$$
\frac{d x}{d t}=c \text { and } \frac{d x}{d t}=-c,
$$

called "characteristic curves". Since $\alpha(x, t)$ and $\beta(x, t)$ are constant along these curves, $\alpha$ and $\beta$ are called the Riemann invariants (see e.g. [16, Tome II, Chap. 12]).

As it has been done in [2] in the case of an overhead crane with a variable length flexible cable, it is interesting to apply the following change of variable

$$
x=L \sigma
$$

to transform the system into one having a fixed spatial domain for $\sigma$, i.e. $\sigma \in[0,1]$.

According to (13), if we denote :

$$
\left\{\begin{array}{l}
\tilde{\alpha}(\sigma, t)=\alpha(x, t)=\alpha(L(t) \sigma, t) \\
\tilde{\beta}(\sigma, t)=\beta(x, t)=\beta(L(t) \sigma, t)
\end{array}\right.
$$

equations (10) and (11) become : 


$$
\left\{\begin{array}{l}
\frac{\partial \tilde{\alpha}}{\partial t}(\sigma, t)+\left(\frac{c-\dot{L} \sigma}{L}\right) \frac{\partial \tilde{\alpha}}{\partial \sigma}(\sigma, t)=0 \\
\frac{\partial \tilde{\beta}}{\partial t}(\sigma, t)-\left(\frac{c+\dot{L} \sigma}{L}\right) \frac{\partial \tilde{\beta}}{\partial \sigma}(\sigma, t)=0 .
\end{array}\right.
$$

We still have two wave equations, but with time variable velocities depending on $L$ and on the control variable $\dot{L}$, where $\dot{L}$ denotes the time derivative of $L$.

Remark 1: The term due to the piston velocity, should be interpreted as acoustic source terms when placed at the right hand side of equation (15). Nevertheless, the velocity of the waves equations remain time dependent through $L$ and the explicit expression of $\tilde{\alpha}$ and $\tilde{\beta}$ become rather complicated to derive through the associated characteristic equations and the boundary conditions.

\section{PhysicAl MOdels OF THE JET CHANNEL AND THE} MOUTH

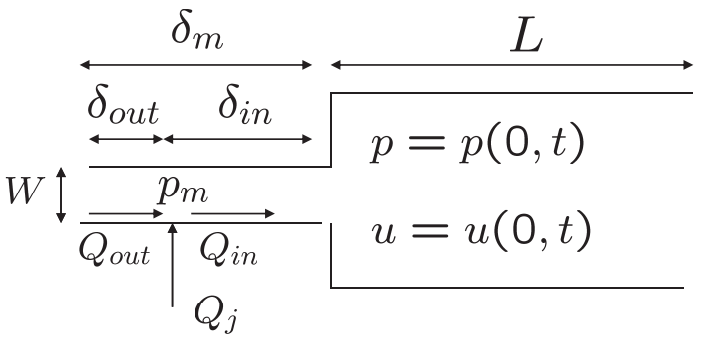

Fig. 2. The 1D model of the mouth

In [18], [20], [19], [9], the two-dimensional geometry of the mouth is modeled in a low frequency plane wave approximation by a one-dimensional representation, by an equivalent pipe segment of length $\delta_{m}$ (see Fig. 2) taking into account the constriction of the pipe at the blowing end.

In this one-dimensional representation, the flue exit, when the jet is formed, is located at an acoustic distance $\delta_{\text {out }}$ from the outside and $\delta_{i n}$ from the entrance of the resonator.

At the flue exit, because the region is compact, one can apply the mass conservation law :

$$
Q_{j}+Q_{\text {out }}=Q_{\text {in }}
$$

where $Q_{j}, Q_{\text {out }}$ and $Q_{i n}$ are respectively the jet flow, the flow in the portion $\delta_{\text {out }}$ and $\delta_{\text {in }}$ respectively, expressed in $\mathrm{m}^{3} / \mathrm{s}$.

The pressure $p_{m}$ in the mouth at the flue exit can be related to the flow $Q_{\text {out }}$ by the radiation impedance, which leads in the time domain to the following linear differential equation :

$$
\left\{\begin{array}{l}
p_{m}=c_{2} \ddot{Q}_{\text {out }}-c_{3} \dot{Q}_{\text {out }} \\
c_{2}=\frac{\rho_{0} r_{m}^{2}}{4 c S_{m}} \text { and } c_{3}=\frac{\rho_{0} \delta_{\text {out }}}{S_{m}}
\end{array}\right.
$$

where $S_{m}$ is the mouth cross section at the flue exit, and $r_{m}$ is the radius of a circle having the same mouth cross section, i.e. such that $\pi r_{m}^{2}=S_{m}$.
Neglecting friction, the jet at the flue exit is governed by the Bernoulli equation :

$$
\rho_{0} l_{c} \frac{d U_{j}}{d t}+\frac{1}{2} \rho_{0} U_{j}^{2}=p_{f}-p_{m}
$$

where $U_{j}$ denotes the jet velocity in the flue channel, $l_{c}$ the length of the channel, $p_{f}$ denotes the excitation pressure at the entrance of the channel, generated by the player and $p_{m}$ denotes the pressure in the mouth of the instrument (see Fig. 1).

Noticing that the flow continuity is assumed at the entrance of the resonator, that is : $Q_{i n}(0)=u(x=0, t)=u_{0}(t)$, the pressure $p(x=0, t)=p_{0}(t)$ can also be related to the pressure $p_{m}$ through momentum conservation :

$$
\left\{\begin{array}{l}
p_{m}-p_{0}=c_{1} \dot{u}_{0}-\Delta p \\
c_{1}=\frac{\rho \delta_{i n}}{S_{m}}
\end{array}\right.
$$

where $\Delta p$ represents the pressure jump across the pipe of length $\delta_{i n}$. This pressure jump, responsible for the sound production, can be mainly decomposed in two terms :

$$
\Delta p=\Delta p_{j d}+\Delta p_{a}
$$

$\Delta p_{j d}$ denoting the pressure jump due to the jet drive mechanism and $\Delta p_{a}$ the vortex shedding when the flow separates at the edge of the labium which appears to be determinant in limiting the amplitude of the oscillation during steady-state, but also to be important to describe nonlinear behavior in the transient attack.

\section{The term $\Delta p_{j d}$}

As explained for example in [20], [19], the pressure due to the jet-drive is determined by the time derivative of the flow source $Q_{1}$ corresponding to the portion of the jet flow entering the pipe at the labium :

$$
\Delta p_{j d}=-\frac{\rho_{0} \delta_{d}}{S_{m}} \frac{d Q_{1}}{d t}
$$

where $\delta_{d}$ is the acoustic distance between the dipole sources $Q_{1}$ and $Q_{2}$ generated at the labium, in the one-dimensional representation of the instrument. Assuming the jet has a Bickley velocity profile (see e.g. [14]), denoting $H$ the jet width, $y_{0}$ the labium position with respect to the flue exit axis ( $y$ being positive towards the interior of the pipe) the following expression is obtained :

$$
Q_{1}=b H \bar{U}_{j}\left[1+\tanh \left(\frac{\eta-y_{0}}{b}\right)\right]
$$

where $b$ is a positive jet parameter characterizing the velocity profile at the flue exit, $\eta$ denotes the jet position which will be detailed in the next paragraph and $\bar{U}_{j}$ denotes the asymptotic value of the jet velocity depending on the excitation pressure $p_{f}$ (see equation (18)), i.e. :

$$
\bar{U}_{j}=\sqrt{2 p_{f} / \rho_{0}} .
$$




\section{The term $\Delta p_{a}$}

Using e.g. [10], [20], [19], one can express the vortex shedding term induced at the labium by the transverse acoustic flow of the pipe by the following expression :

$$
\Delta p_{a}=-\frac{1}{2} \rho_{0}\left(\frac{u_{0}}{\alpha_{v} S_{m}}\right)^{2} \operatorname{sign}\left(u_{0}\right)
$$

where $\alpha_{v}$ is the vena-contracta factor of the flow. It can be seen that this term is dissipative, corresponding to the kinetic energy dissipation by turbulence of the jet, formed by separation of the acoustic flow at the labium.

\section{Physical model of the jet position}

Let us now give an expression of the jet position $\eta$ in the mouth obtained from recent works e.g. [8], denoting $h$ the jet height :

$$
\eta(t)=2 \frac{u_{0}\left(t-\tau_{l}\right) h}{\pi S_{m} U_{j}} e^{\mu W}
$$

where $\mu$ denotes the spatial amplification of the jet, $W$ the distance between the flue exit and the labium and the delay $\tau_{l}$ is given by :

$$
\tau_{l}=\frac{W}{0.3 U_{j}} .
$$

We can see that the delay $\tau_{l}$ is time varying since it depends on $U_{j}$. But, in the transient regime of the jet velocity, $U_{j}$ takes values near the origin, so that in numerical simulations, we have to wait $U_{j} \geq \gamma$ for a small positive value $\gamma$ to consider equation (26). Before that, we take $\eta=0$.

\section{BOUNDARY CONDITIONS}

Let us now complete the pipe model (15) with the boundary conditions at $\sigma=0$ (i.e. $x=0$ ) and $\sigma=1$ (i.e. $x=L$ ).

\section{A. Boundary condition at the entrance of the resonator}

Let us first consider the boundary condition at the entrance of the resonator. It can be obtained replacing $p_{m}$ from equation (19) in equation (18), which leads to :

$$
p_{0}(t)=p_{f}-\rho_{0} l_{c} \frac{d U_{j}}{d t}-\frac{1}{2} \rho_{0} U_{j}^{2}-c_{1} \dot{u}_{0}(t)+\Delta p .
$$

This boundary condition can be rewritten in the $\alpha$ and $\beta$ variables using (9) and in the $\tilde{\alpha}$ and $\tilde{\beta}$ variables, using (14) which gives finally :

$$
\begin{aligned}
& \tilde{\alpha}(0, t)=\tilde{\beta}(0, t)+ \\
& \frac{2 S_{p}}{\rho_{0} c}\left[p_{f}-\rho_{0} l_{c} \frac{d U_{j}}{d t}-\frac{1}{2} \rho_{0} U_{j}^{2}-\frac{c_{1}}{2}(\dot{\tilde{\alpha}}+\dot{\tilde{\beta}})(0, t)+\Delta p\right]_{\text {(28) }}
\end{aligned}
$$

Remark 2: In the previous paper [3], the boundary condition which was used was the very simple one $p(x=0, t)=$ 0 , i.e. $\tilde{\alpha}(0, t)=\tilde{\beta}(0, t)$, corresponding to an ideal case. Taking into account the physical models of the jet and of the mouth leads to the more realistic above condition. It can be also noticed that $p_{0}(t)$ now depends on $\dot{u}_{0}(t)$ but using (20), (21), (22), (24) and (25) also on $u_{0}(t)$ and $\dot{u}_{0}\left(t-\tau_{l}\right)$.
Moreover, we need the value of $U_{j}$ and its time-derivative to bring up to date the boundary condition (28). So we have to solve at each time instant, the ordinary differential equation describing the dynamical evolution of $U_{j}$. This equation is obtained from (18) where we replace $p_{m}$ by its expression (17) and using equation (16) which becomes at $x=0$ :

$$
Q_{\text {out }}=Q_{\text {in }}-Q_{j}=u_{0}(t)-S_{e} U_{j}
$$

$S_{e}$ denoting the cross section of the channel at the flue exit.

Finally, the equation giving the value of $U_{j}(t)$ can be written :

$$
c_{2} S_{e} \ddot{U}_{j}-\left(\rho_{0} l_{c}+c_{3} S_{e}\right) \dot{U}_{j}+c_{3} \dot{u}_{0}-c_{2} \ddot{u}_{0}=\frac{1}{2} \rho_{0} U_{j}^{2}-p_{f} .
$$

When taking realistic numerical values of the constants involved in (30) it can be seen that $c_{2} S_{e} \simeq 10^{-9}$, which is negligible with respect to the multiplying factor of $\dot{U}_{j}$. Therefore, using singular perturbation arguments, one can neglect the terms in $\ddot{U}_{j}$ in (30) and we can consider the following equation which will be used to evaluate $U_{j}$ and its time derivative :

$$
\left(\rho_{0} l_{c}+c_{3} S_{e}\right) \dot{U}_{j}=p_{f}-\frac{1}{2} \rho_{0} U_{j}^{2}+c_{3} \dot{u}_{0}-c_{2} \ddot{u}_{0} .
$$

Finally, the boundary condition at the entrance of the resonator consists in the two equations (28) and (31) at $x=0$.

\section{B. Boundary condition at the end of the resonator}

Considering the piston mechanism which allows the translation of the slide flute, the boundary condition at the end of the flute, can be written as a first approximation neglecting friction terms :

$$
S_{p} p(L, t)+F=m \ddot{L}
$$

$F$ being the force exerted by the motor on the slide and $m$ the piston mass.

In a first step, one can consider that the control variable is the piston velocity $\dot{L}$, linked to the physical control $F$ homogeneous to $\ddot{L}$, via the integrator (or cascade) system given by (32). Then if $\dot{L}$ is known, one can then compute the physical control $F$ to apply, using e.g. "backstepping" techniques (see [1]). One can therefore consider, without loss of generality, the following boundary condition at $x=L$ :

$$
u(L, t)=S_{p} \dot{L}
$$

which can be rewritten in the $\tilde{\alpha}$ and $\tilde{\beta}$ variables, using (9) and (14) :

$$
\tilde{\alpha}(1, t)+\tilde{\beta}(1, t)=2 S_{p} \dot{L} .
$$




\section{MOdAl ANALYSis OF THE LINEARIZED BOUNDARY CONDITIONS}

To compute the natural modes of the system, we have to keep only linear terms in the boundary conditions obtained in the previous section. More precisely, we approximate tanh by its argument in (22) for the expression of $\Delta p_{j d}$ and we neglect the nonlinear term $\Delta p_{a}$ given by (24). Writing $U_{j}$, $\tilde{\alpha}$ and $\tilde{\beta}$ on the following form, where $\bar{U}_{j}$ is the steady state value of the jet velocity (see equation (23)) :

$$
\left\{\begin{array}{l}
U_{j}=\bar{U}_{j}+U e^{i \omega t} \\
\tilde{\alpha}(x, t)=a e^{i \omega t} e^{-i \omega x / c} \\
\tilde{\beta}(x, t)=b e^{i \omega t} e^{i \omega x / c}
\end{array}\right.
$$

replacing $U_{j}, \tilde{\alpha}$ and $\tilde{\beta}$ in (28) and (31) at $x=0$ and (34) at $x=L$ and keeping only linear terms, we have to solve an homogeneous linear system of 3 equations with 3 complex unknowns $(U, a, b)$ of the form, where denoting $\kappa(\omega)=\left(c_{1}+\bar{K} e^{-i \omega \tau_{l}}\right) i \omega$ :

$$
\begin{aligned}
& A(\omega)\left(\begin{array}{c}
a \\
b \\
U
\end{array}\right)=0, \text { with } A(\omega)= \\
& \left(\begin{array}{lll}
Z_{c}+\kappa(\omega) & -Z_{c}+\kappa(\omega) & 2 \rho_{0}\left(\bar{U}_{j}+i \omega l_{c}\right) \\
c_{3} i \omega+c_{2} \omega^{2} & c_{3} i \omega+c_{2} \omega^{2} & -2\left(\rho_{0} \bar{U}_{j}+i \omega c_{0}\right) \\
e^{-i \omega L / c} & e^{i \omega L / c} & 0
\end{array}\right)
\end{aligned}
$$

where the constants $c_{1}, c_{2}$ and $c_{3}$ are given by (19) and (17), the characteristic impedance $Z_{c}$ is given by :

$$
Z_{c}=\frac{\rho_{0} c}{S_{p}},
$$

and the constant $\bar{K}$ is obtained by linearizing $\Delta p_{j d}$, the pressure jump due to the jet-drive (see equations (21), (22) and (25)). In fact, after some computations, we obtain the linearized expression $\Delta \bar{p}_{j d}$ :

$$
\left\{\begin{array}{l}
\overline{\Delta p_{j d}}=\bar{K} \dot{u}_{0}\left(t-\tau_{l}\right) \\
\bar{K}=\frac{2 \rho_{0} \delta_{d} h e^{\mu W} H}{\pi S_{m}^{2}}\left(1-y_{0}^{2} / b^{2}\right) .
\end{array}\right.
$$

Therefore, to obtain a non trivial solution, the complex modes $\omega=\lambda+i \epsilon$ must satisfy :

$$
\operatorname{det}(A(\omega))=0
$$

namely, the real numbers $\lambda$ and $\epsilon$ must be solutions of the two real equations :

$$
\left\{\begin{array}{l}
\operatorname{Re}(\operatorname{det}(A(\lambda, \epsilon)))=0 \\
\operatorname{Im}(\operatorname{det}(A(\lambda, \epsilon)))=0
\end{array}\right.
$$

In Fig. 3, we can see the 2D-surfaces $\operatorname{Re}(\operatorname{det}(A(\lambda, \epsilon)))=0$ and $\operatorname{Im}(\operatorname{det}(A(\lambda, \epsilon)))=0$ parameterized by $\lambda$ and $\epsilon$.

For $L=0.265 \mathrm{~m}$ and $p_{f}=300 \mathrm{~Pa}$, the three first frequencies $f_{i_{c}}, i=1,2,3$, corresponding to the three first modes $\lambda_{i_{c}}=2 \pi f_{i_{c}}, i=1,2,3$ are approximately, in $H z$ :

$$
f_{1_{c}} \simeq 290.8, f_{2_{c}} \simeq 903.7, f_{3_{c}} \simeq 1474 .
$$

The corresponding values of $\epsilon_{i_{c}}$ are, in $s^{-1}$ :

$$
\epsilon_{1_{c}} \simeq 36.9, \epsilon_{2_{c}} \simeq-24.2, \epsilon_{3_{c}} \simeq-74.7 .
$$
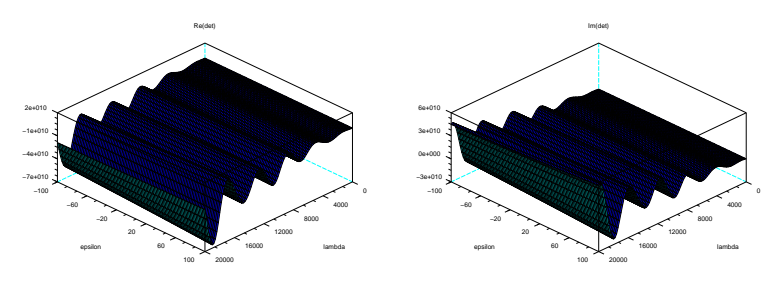

Fig. 3. $\operatorname{Re}(\operatorname{det}(\mathrm{A}))$ and $\operatorname{Im}(\operatorname{det}(\mathrm{A}))$ with respect to $\lambda$ and $\epsilon$

It can be noticed that :

$$
\frac{f_{2_{c}}}{f_{1_{c}}} \simeq 3.11, \frac{f_{3_{c}}}{f_{1_{c}}} \simeq 5.07
$$

i.e. the frequencies of the modes are close to odd multiples of the first mode frequency, as expected for a closed-open pipe. For negative values of $\epsilon$, the modes are oscillating corresponding to a time growth. Since they are solutions of the linearized problem, the modes calculated here can predict the oscillating frequency during transient state, at low amplitude. After this initial period, the saturation mechanisms may be responsible for a bifurcation in the system.

\section{Vi. Automatic control of the flute}

To realize an automatic control law, we have to compute $p_{f}$ and $L$ or equivalently, the pair $\left(\bar{U}_{j}, L\right)$ such that a desired reference mode $\omega_{r}=\lambda_{r}+i \epsilon_{r}$ (or a desired reference frequency $f_{r}=\frac{\lambda_{r}}{2 \pi}$ ) is obtained. Therefore, the control algorithm can be summarized as follows :

- $\lambda_{r}$ and $\epsilon_{r}$ being chosen, solve the two equations (40) with respect to the unknown variables $\bar{U}_{j r}$ and $L_{r}$, using for example a Newton algorithm.

- The resulting asymptotic jet velocity $\bar{U}_{j r}$ will be reached through the servo-valve, asking for a desired steady-state pressure $p_{f_{r}}=\frac{1}{2} \rho_{0} \bar{U}_{j r}^{2}$.

- The resulting length of the pipe $L_{r}$ will be reached, applying the following simple linear control law on the piston :

$$
\dot{L}=-k\left(L-L_{r}\right), k>0
$$

For example, to have $\omega_{r}=2039$, corresponding to $f_{r}=f_{1_{\text {ideal }}}=c / 4 \mathrm{~L}=324 \mathrm{~Hz}$, solving equations (40) with initial conditions close to the first mode frequency leads to : $\left(\bar{U}_{j r}=8.61 \mathrm{~m} / \mathrm{s} ; L_{r}=0.242\right)$ (or equivalently $\left.\left(p_{f_{r}}=44.5 \mathrm{~Pa} ; L_{r}=0.242\right)\right)$.

\section{NUMERICAL SCHEME, SIMULATION RESULTS AND CONCLUSION}

The numerical model which will be used for simulation and control is obtained by (15) together with the boundary conditions (28), (34) and (31). Since the velocities are respectively positive and negative for $\tilde{\alpha}$ and $\tilde{\beta}$, it was natural to implement a first-order un-centered upper scheme for $\tilde{\alpha}$ and down scheme for $\tilde{\beta}$. Numerical diffusion terms should be added to use centered schemes (see e.g. [15]). 
To be more realistic and take into account viscous and thermal losses in the tube, we have also added a small friction term of the form $\nu u$ in Euler equation (1), $\nu$ being a small friction coefficient. More accurate models exist for viscothermal damping using non integer time derivative of the flow rate (see e.g. [13]).

In Fig. 4 and 5, we can observe two spectrograms of the simulated pressure. For comparison, the set point blowing pressure used in the simulation is the one measured on the experimental setup described below for pressures close respectively to $55 \mathrm{~Pa}$ and $245 \mathrm{~Pa}$.

The spectrogram of the pressure at $x=0$ in Fig. 5 when $p_{f} \simeq 245 \mathrm{~Pa}$ shows that the oscillation takes place on the second acoustic mode of the pipe $f_{2_{s}} \simeq 925 \mathrm{~Hz}$ which is very close to the computed values $f_{2 c}$ given in (41). For lower values of the blowing pressure, for example $p_{f}=55 \mathrm{~Pa}$ in Fig. 4, simulations show an oscillation on the first pipe mode around $310 \mathrm{~Hz}$ also close to $f_{1_{c}}$ given in (41). The slight difference is probably due to the fact that the computed frequencies $f_{i_{c}}$ are obtained through linearized boundary conditions. Nevertheless, equations (40) constitute a good approximation to compute $L_{r}$ as explained in section VI.

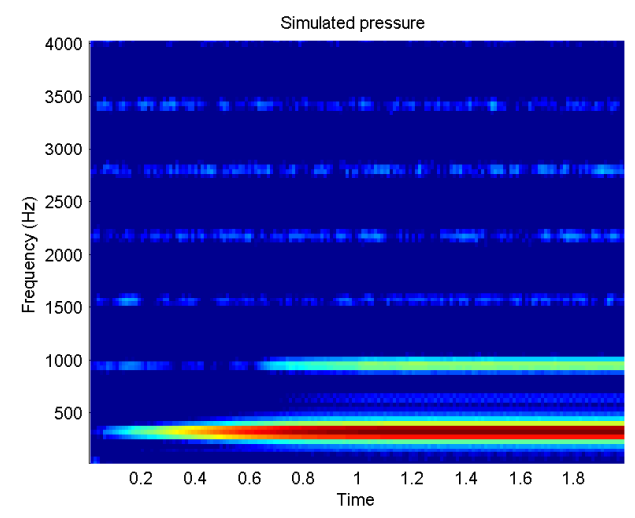

Fig. 4. Spectrogram of the simulated pressure using the experimental blowing pressure of Fig. 6 (close to $55 \mathrm{~Pa}$ ) and $L=0.265 \mathrm{~m}$

The resulting pressure is now compared with experimental data obtained from an actual instrument. This instrument is built on a $18.4 \mathrm{~mm}$ diameter tube, associated with a recorderlike mouth piece. The tube is closed at its passive end. The total length of the pipe is $265 \mathrm{~mm}$. The window shows lateral walls of about $4 \mathrm{~mm}$ which act as ears in an organ pipe, increasing the end correction associated with radiation. The attack transient is generated by a pressure rise time of about $40 \mathrm{~ms}$ and shows an overshoot. The acoustic pressure in the instrument is measured at $16 \mathrm{~mm}$ of the block of the flute. From Fig. 6 and 7, one can observe that the oscillation regimes for $p_{f} \simeq 55 \mathrm{~Pa}$ and $p_{f} \simeq 245 \mathrm{~Pa}$ are the same as in the simulated cases (see Fig. 4 and 5). In the case $p_{f} \simeq 55 P a$, the experimental spectrum during steady state is

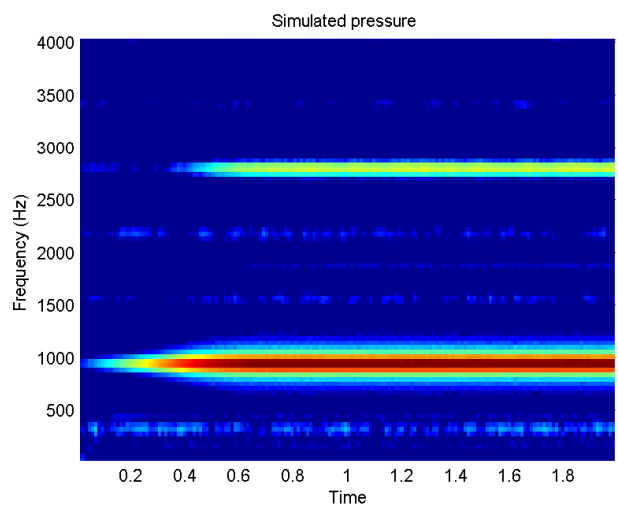

Fig. 5. Spectrogram of the simulated pressure using a higher experimental blowing pressure of Fig. 7 (close to $245 \mathrm{~Pa}$ ) and $L=0.265 \mathrm{~m}$

dominated by harmonics 1 and 3 whereas the even harmonics appear weakly. These even harmonics are generated by nonlinear source terms (see equations (22)-(24)). They are weak compared to odd harmonics because they do not benefit of pipe resonances. For all flute-type instruments, increasing the blowing pressure results in a transition to an upper mode. In the case of a stopped pipe, the second mode is approximately at frequency 3 times higher than the first mode. Therefore, as expected in the case of overblowing pressure $p_{f} \simeq 245 \mathrm{~Pa}$, the oscillating regime is given by the second mode, both in experimental and simulated cases. The experimental pressure also shows noise modulated by the passive resonances of the pipe. This noise is generated by turbulence as discussed in [18]. This phenomenon has not been taken into account in our simulation model.

During the steady-state part of the oscillation, the simulation and experimental pressure signals show some similarities but also some differences. The oscillation regime is the same for simulated and measured systems : oscillation on the first pipe mode for $p_{f} \simeq 55 \mathrm{~Pa}$ and oscillation on the second pipe mode for $p_{f} \simeq 245 \mathrm{~Pa}$. The main differences are due to the fact that the experimental flute presents inharmonicity that the simulated one does not include. This is due to the dispersion associated with viscous and thermal effects at the pipe walls, which have not been included in our model, and also to the fact that the frequency dependence of the end corrections has not be modeled. The amplitude of the even harmonics appears to be relatively higher in the experimental data than in the simulation. This can be interpreted in terms of the simplification of the non-linear source terms in the model but also of the turbulent sound production already discussed.

Moreover, we can observe on Fig. 8 that the amplitude of the pressure oscillation predicted by the simulation is close to the one observed in the experimental data. This indicates that the dissipation model is quite efficient despite its simplicity.

The oscillation starts more slowly in the simulated flute than in the experimental one. Indeed the jet and source model 


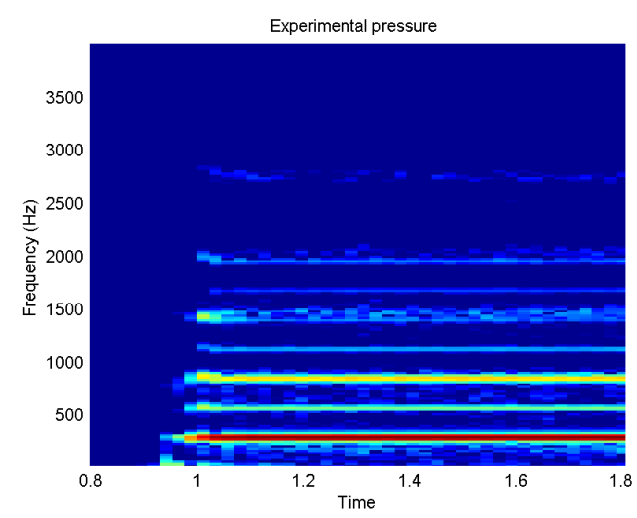

Fig. 6. Spectrogram of the experimental pressure with $p f$ close to $55 \mathrm{~Pa}$ and $L=0.265 \mathrm{~m}$

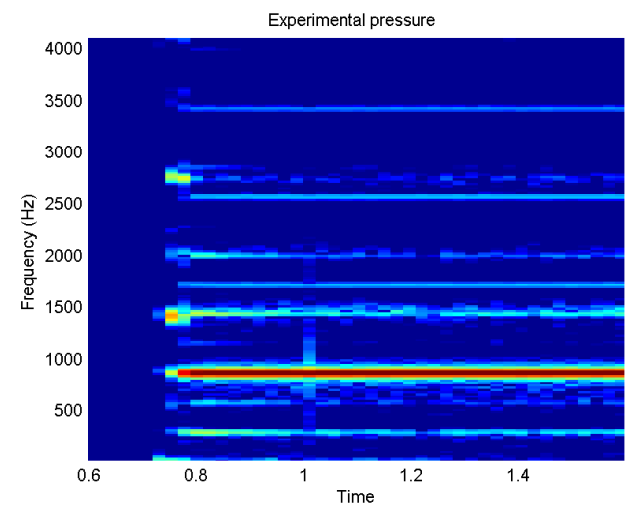

Fig. 7. Spectrogram of the experimental pressure with $p_{f}$ close to $245 \mathrm{~Pa}$ and $L=0.265 \mathrm{~m}$

we use are based on the analysis of steady-state oscillations. Some aspects of the starting transient are not yet well described by such models, as discussed in [9]. Moreover, these models have been elaborated for open recorder-like instruments and should be adapted in the case of a stopped pipe, but this work is out of the scope of the present paper.
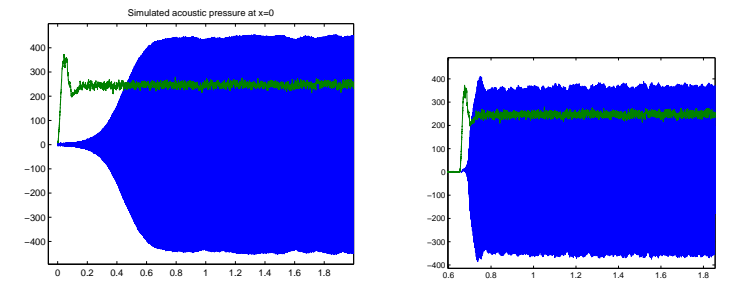

Fig. 8. Comparison between the model pressure and the experimental pressure with the same excitation blowing pressure $p_{f} \simeq 245 \mathrm{~Pa}$

As we have already said in the introduction, the blowing pressure range obtained for example in [4] or [5, Sec. 3] was lower than in actual pipes. This is mainly due to the simplifications of the jet and source models (eqs. (25), (26) and (21)) which overestimate the jet amplification and sound production at low jet velocities. To solve this problem, we have implemented a numerical first order low pass filter on the jet position $\eta$ leading to $\eta_{f}$ to obtain a more realistic dynamics of the jet position in the mouth :

$$
\eta_{f}(k+1)=c_{4} \eta_{f}(k)+\left(1-c_{4}\right) \eta(k), c_{4} \in[0 ; 1[.
$$

Finally, Fig. 9 displays the simulation results we obtain when the player wants to produce scales by sliding the piston. It can be seen that the transient time response is about $0.1 \mathrm{~s}$ which is coherent with the attack transient time observed when the slide is fixed. The spectrogram shows the frequency shift resulting from the piston sliding.
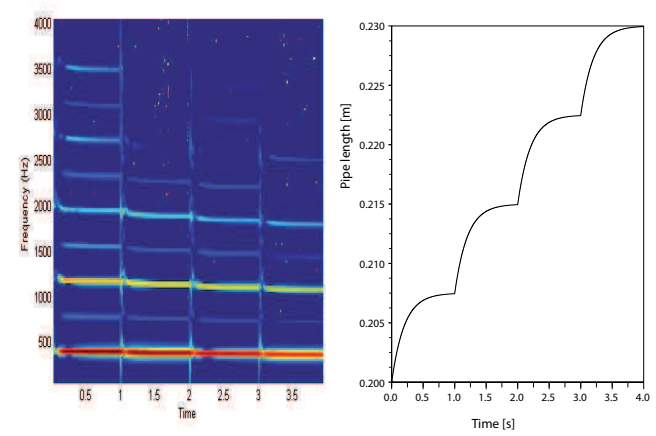

Fig. 9. A down scale : on the left spectrogram of the acoustic pressure; on the right evolution of $L$ from $0.20 \mathrm{~m}$ to $0.23 \mathrm{~m}$

To conclude, the model presented in this paper produces satisfying simulation results and could be used for sound synthesis. Concerning the control, it must be noticed that the $\left(p_{f} ; L\right)$ solution to reach a target pitch is not unique. Indeed, the slide flute can play the same pitch with a soft blowing and a short slide position, or with an increased blowing pressure and a longer slide position. The instrument therefore can produce musical dynamics (piano e forte) at constant pitch by adjusting both the blowing pressure and the slide position. The $\left(p_{f} ; L\right)$ solution presented at the end of section VI corresponds to the closest solution to the $L$ value for passive resonance.

An experimental prototype is under development at "Mines ParisTech" as shown in Fig. 10. The reference values $\left(p_{f_{r}} ; L_{r}\right)$ have been obtained from the modal analysis performed on the linearized boundary conditions. For musical 
applications, the target is defined as a pitch rather than a length. Consequently, it is more natural to regulate the frequency error and this necessitates to implement a real-time frequency detection to elaborate the closed-loop control.

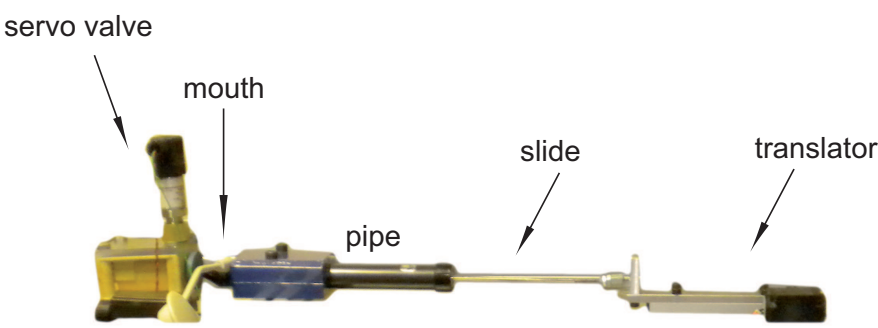

Fig. 10. The slide flute prototype

\section{REFERENCES}

[1] B.d'Andréa-Novel, J.-M. Coron, Exponential stabilization of an overhead crane with flexible cable via a back-stepping approach, Automatica, 36, pp. 587-593, (2000).

[2] B. d'Andréa-Novel, J.-M. Coron, Stabilization of an overhead crane with a variable length flexible cable, Computational and Applied Mathematics, Vol. 21, No. 1, pp. 101-134, (2002).

[3] B. d'Andréa-Novel, J.-M. Coron, G. Bastin, Sur le contrôle frontière d'une flûte à coulisse par fonction de Lyapunov, e-STA : Numéro spécial CIFA 2006, Vol. 4 No. 1 Commande : aspects méthodologiques et applicatifs, Janvier 2007.

[4] B. d'Andréa-Novel, B. Fabre, J.-M. Coron, Modeling and control of a slide flute. Proc. of 16th Mediterranean Conference on Control and Automation, pp. 1568-1573, Ajaccio, France, June 2008.

[5] B. d'Andréa-Novel, J.-M. Coron, B. Fabre, T. Hélie, Wind instruments as time delay systems. Part I : modeling, Proc. of TDS Conference, Sinaia, Romania, September 2009.

[6] J.W. Coltman, Jet drive mechanism in edge tones and organ pipes, J. Acoust. Soc. Am. 60, (3), 1976, pp. 725-733.

[7] L. Cremer, H. Ising, Die selbsterregten Schwingungen von Orgelpfeifen, Acustica, 19, 1968, pp. 143-153.

[8] P. de la Cuadra, The sound of oscillating air jets : physics, modeling and simulation in flute-like instruments, Ph.D. thesis, Stanford University, December 2005.

[9] B. Fabre, A. Hirschberg, Physical modeling of flue instruments : a review of lumped models, Acta Acustica 86, 2000, pp. 599-610.

[10] B. Fabre, A. Hirschberg, A.P.J. Wijnands, Vortex shedding in steady oscillations of a flue organ pipe, Acustica 82, 1996, pp. 863-877.

[11] N.H. Fletcher, Sound production by organ flue pipes, J. Acoust. Soc. Am., 60 (4), 1976, pp. 926-936.

[12] M.S. Howe, Contribution to the theory of aerodynamics sound, with application to excess jet noise and the theory of the flute, J. Fluid Mech., 71, 1975, pp. 625-673.

[13] D. Matignon, Représentation en variables d'état de modèles de guide d'ondes avec dérivation fractionnaire, $\mathrm{PhD}$ Thesis, Université de Paris-Sud, Orsay, France, 1994.

[14] A. Powell, On edge tones and associated phenomena, Acustica 3, 1953, pp. 233-243.
[15] L. Sainsaulieu, Calcul scientifique, Masson, Paris, 1996.

[16] D. Serre, Systèmes de lois de conservation, Diderot Editeur, Arts et Science, Paris, 1996.

[17] M.P. Verge, B. Fabre, W.E.A. Mahu, A. Hirschberg, R. Van Hassel, A.P.J. Wijnands, J.J. de Vries, C.J. Hogendoorn, Jet formation and jet velocity fluctuations in a flue organ pipe, J. Acoust. Soc. Am., 95 (2), 1994, pp. 1119-1132.

[18] M.-P. Verge, Aeroacoustics of confined jets, with applications to the physical modeling of recorder-like instruments, Ph.D. thesis, Technische Universiteit Eindhoven, October 1995.

[19] M.-P. Verge, A. Hirschberg, R. Caussé, Sound production in recorderlike instruments. A simulation model, J. Acoust. Society. Am., Vol. 101(5), 1997, pp. 2925-2939.

[20] M.-P. Verge, R. Caussé, B. Fabre, A. Hirschberg, A.P.J. Wijnands, A. van Steenbergen, Jet oscillations and jet drive in recorder-like instruments, Acustica 2, 1994, pp. 403-419. 